\title{
ML Symbol Synchronization for OFDM-based WLANs in Unknown Frequency-Selective Fading Channels
}

\author{
Yik-Chung $\mathrm{Wu}^{*}$, Kun-Wah Yip ${ }^{\dagger}$, Tung-Sang $\mathrm{Ng}^{\ddagger}$ and Erchin Serpedin* \\ *Department of EE, Texas A\&M University, College Station, TX 77843-3128, USA. \\ ${ }^{\dagger}$ Philips Research East Asia - Shanghai, China. \\ ${ }^{\ddagger}$ Department of EEE, The University of Hong Kong, Pokfulam Road, Hong Kong.
}

\begin{abstract}
Based on the maximum-likelihood principle and the preamble structure of IEEE 802.11a WLAN standard, this paper proposes a new symbol synchronization algorithm for IEEE 802.11a WLANs over frequency-selective fading channels. In addition to the physical channel, the effects of filtering and unknown sampling phase offset are also considered. Loss in system performance due to synchronization error is used as a performance criterion. Computer simulations show that the proposed algorithm has comparable performances to the algorithm based on the generalized Akaike information criterion (GAIC), but the proposed algorithm exhibits reduced complexity.
\end{abstract}

\section{INTRODUCTION}

IEEE 802.11a wireless local area networks (WLANs), which support high-speed data transmissions up to $54 \mathrm{Mbps}$ [1], employ burst-mode transmission and orthogonal frequency division multiplexing (OFDM) as the transmission technique. Although OFDM is well known for its ability to combat the intersymbol interference (ISI) introduced by multipath channels [2], [3], incorrect positioning of the FFT window within an OFDM symbol reintroduces ISI during data demodulation, causing serious performance degradation [4], [5]. Symbol synchronization is therefore one of important tasks performed at receivers in IEEE 802.11a WLANs.

A number of methods for OFDM symbol synchronization have been proposed in the literature (e.g., methods exploit the periodic structure of cyclic prefixes in OFDM symbols [5][7] and algorithms based on the use of repeated preambles [8]-[12]). Although the techniques of [4]-[12], which were originally developed for general OFDM systems, may be applied to IEEE 802.11a WLANs, a higher synchronization accuracy can be obtained by using optimized algorithms that take advantage of the known preamble structure located at the beginning of a data packet.

Recently, symbol synchronization techniques that are specifically designed for IEEE 802.11a WLANs have been reported in [13] and [14]. In [13], the received signal is correlated with a known training-symbol sequence and the absence of the expected correlation peak is detected. Despite the advantage that a simple correlator can be easily implemented at the receiver, its performance is poor in dispersive channels [13], indicating that more sophisticated synchronization algorithms are required. In [14], the generalized Akaike information criterion (GAIC) is used to jointly estimate the channel and establish timing synchronization. Although the reported performance is good, its complexity is extremely high.

In this paper we develop a maximum-likelihood (ML) symbol synchronizer for IEEE 802.11a WLANs on frequencyselective fading channels. A realistic channel, which includes the effects of filtering and sampling time offset in addition to the physical channel with random path delays, is considered. Furthermore, the loss in system performance due to synchronization error is used as the performance criterion [5], [19], as opposed to the requirement that the estimated symbol timing has to be within certain limits with respect to a fixed reference point. Simulation results indicate that the proposed algorithm has comparable performances to the algorithm based on GAIC [14], but the complexity of the proposed algorithm is much smaller than that of GAIC algorithm due to the smaller observation length.

\section{RECEIVED-SIGNAL MODEL}

\section{A. Signal and Channel Models}

Fig. 1 depicts the packet structure used in IEEE 802.11a WLANs. In each packet, the data carrying OFDM symbols are preceded by a preamble, which is used for start of packet detection, automatic gain control, symbol timing and frequency synchronization, and channel estimation. The preamble itself consists of two parts. The first part comprises 10 short training symbols, $b(t)$, each of length $T_{b}=800 \mathrm{~ns}$. In the second part, a cyclic prefix, $g(t)$, of length $T_{g}=1.6 \mu \mathrm{s}$ is followed by two long training symbols, $c(t)$, each of length $T_{c}=3.2 \mu \mathrm{s}$.

Let the baseband-equivalent signal of the preamble be $s(t)$ (the equations for $b(t), g(t)$ and $c(t)$ can be found in the standard [1] and are not detailed here). The signal $s(t)$ is passed through the transmission filter, up-converted to high frequency and transmitted through a multipath frequencyselective fading channel. At the receiver, the signal is downconverted into baseband signal and then passed through receiving filter. Assuming the channel is static over the duration of a packet, the complex envelope of the received and filtered signal is given by

$$
r(t)=\int_{-\infty}^{\infty} s(t-u)\left[\sum_{n=0}^{L_{o}-1} \gamma_{n} f\left(u-\tau_{n}\right)\right] d u+w(t) .
$$


where $\gamma_{n}$ is the complex valued channel coefficients for the $n^{\text {th }}$ path with $\tau_{n}$ as the path delay, $L_{o}$ is the total number of physical path of the multipath channel, $f(t)$ is the combined response of the transmit and receiving filter, and $w(t)$ is the filtered complex-Gaussian noise. It is assumed that the channel gain is unity (i.e., $\sum_{n=0}^{L_{o}-1} \mathbb{E}\left[\left|\gamma_{n}\right|^{2}\right]=1$, where $\mathbb{E}[$.] stands for expectation). Furthermore, without loss of generality, it is assumed that $\tau_{0}=0$ since the delay of first path can be translated to a delay in sampling.

Now, the received signal is sampled at $t=k T_{\text {sam }}+\varepsilon_{o} T_{\text {sam }}$, where $1 / T_{\text {sam }}=20 \mathrm{MHz}$, which is the suggested sampling rate in the standard [1], and $\varepsilon_{o} \in[0,1)$ is the unknown time offset induced by the combination of the delay of the first path of the channel and the sampling phase offset. It follows that the sampled signal is given by

$$
r_{k}=\frac{1}{T_{\text {sam }}} \int_{-\infty}^{\infty} s\left(k T_{\text {sam }}-u^{\prime}\right) h\left(u^{\prime}\right) d u^{\prime}+w_{k}
$$

where $r_{k} \triangleq r\left(k T_{\text {sam }}+\varepsilon_{o} T_{\text {sam }}\right), w_{k} \triangleq w\left(k T_{\text {sam }}+\varepsilon_{o} T_{\text {sam }}\right)$, $u^{\prime}=u-\varepsilon_{o} T_{\text {sam }}$ and $h(t)$ is the equivalent channel which includes the effects of the transmission filter, physical channel, receiving filter, the timing delay induced by the delay of the first path of the channel and the sampling phase offset, and is defined as

$$
h(t) \triangleq T_{\text {sam }} \sum_{n=0}^{L_{o}-1} \gamma_{n} f\left(t-\tau_{n}+\varepsilon_{o} T_{\text {sam }}\right) .
$$

Notice that the bandwidth (one-sided baseband) of $s(t)$ is $B W_{s} \approx 8.44 \mathrm{MHz}$ [1], meaning that $B W_{s}<1 / 2 T_{\text {sam }}$. According to [18], if the bandwidth of the equivalent channel $h(t)$ (which is equal to the bandwidth of $f(t)$ ) satisfies $B W_{h}<1 / T_{\text {sam }}-B W_{s}$, then by the equivalence of digital and analog filtering for band-limited signals, the sampled received signal can be expressed as

$$
r_{k}=\sum_{i=-\infty}^{\infty} s\left(k T_{\text {sam }}-i T_{\text {sam }}\right) h\left(i T_{\text {sam }}\right)+w_{k} .
$$

A simple example of $f(t)$ that makes the above bandwidth requirement satisfied is the raised cosine filter with $\beta<0.156$ since it is required that $B W_{h}=(1+\beta) / 2 T_{\text {sam }}<1 / T_{\text {sam }}-$ $B W_{s}$. Without loss of generality, we employ square root raised cosine filters with roll-off factor $\beta=0.1$ for both transmit filter and receiving filter for the rest of the paper. Because the receiving filter is a square root cosine filter, the filtered noise samples are uncorrelated with variance $\sigma_{w}^{2}=\mathbb{E}\left[|w(t)|^{2}\right]$.

Remark 1. For the special case that if 1) the path delays are sample spaced (i.e., $\tau_{n}=n T_{\text {sam }}$ ), 2) the timing delay $\varepsilon_{o}=0$ and 3) $f(t)$ is a raised cosine pulse (normalized with $T_{\text {sam }}$ ) with $\beta<0.156$, then

$$
h\left(i T_{\text {sam }}\right)=\sum_{n=0}^{L_{o}-1} \gamma_{n} \delta(i-n),
$$

since the values of the raised cosine pulse are zero at integer multiples of $T_{\text {sam }}$. This is the system model used in [14], where the physical channel is represented by the commonly used tapped delay line model with equal tap spacing [15, p.795]. Therefore, the channel model considered in this paper is more general than that in [14].

\section{B. Matrix Algebraic Formulations}

From (4), it is apparent that the received samples depend on $h\left(i T_{\text {sam }}\right)$ for $-\infty \leq i \leq \infty$. However, in practice, $h\left(i T_{\text {sam }}\right)$ will have significant values only for a finite range of $i$ since 1) the path delays occur in a finite interval and 2) the value of $f(t)$ becomes very small when $|t|$ is large. An example of $\left|h\left(i T_{\text {sam }}\right)\right|^{2}$ is shown in Fig. 2 for $L_{o}=6$, the first tap of the physical channel has zero delay, other five taps have delay uniformly distributed over the interval $0-300 \mathrm{~ns}, \gamma_{n}$ are independent and identically distributed (i.i.d.), zero-mean, complex Gaussian random variable with variances following the multipath intensity profile $\phi(\tau) \sim e^{-\tau / \tau_{r m s}}$, where $\tau_{r m s}=$ $100 \mathrm{~ns}, \varepsilon_{o}$ is a random variable uniformly distributed in $[0,1)$ and $f(t)$ is given by raised cosine filter (normalized with $\left.T_{\text {sam }}\right)$ with $\beta=0.1$. It can be seen that $h\left(i T_{\text {sam }}\right)$ can be well represented by a sequence with finite length. Therefore, (4) can be approximated by

$$
r_{k} \cong \sum_{i=-L_{1}}^{L_{e}-L_{1}-1} s\left(k T_{\text {sam }}-i T_{\text {sam }}\right) h\left(i T_{\text {sam }}\right)+w_{k}
$$

where $L_{e}$ and $L_{1}$ are the total number of taps and the number of taps for $t<0$ in the approximated equivalent channel, respectively. Note that the above approximation can be made arbitrarily accurate by using large enough values of $L_{e}$ and $L_{1}$.

Let $\mathbf{r}_{n}$ be a received-signal vector with $N$ received samples ${ }^{1}$

$$
\mathbf{r}_{n}=\left[\begin{array}{llll}
r_{n} & r_{n+1} & \ldots & r_{n+N-1}
\end{array}\right]^{T},
$$

where $N=T_{b} / T_{\text {sam }}=16$ is the number of samples over the span of a short training symbol $b(t)$. Let $b_{n}=b\left(n T_{\text {sam }}\right)$ and $g_{n}=g\left(n T_{\text {sam }}\right)$ be the $n^{t h}$ samples of the short training symbol and of the cyclic prefix, respectively. For $L_{e}-L_{1} \leq$ $n \leq 9 N-L_{1}, \mathbf{r}_{n}$ is given by

$$
\mathbf{r}_{n}=\mathbf{B}_{n+L_{1}}^{\left(L_{e}\right)} \mathbf{h}_{o}+\mathbf{w}_{n},
$$

where

$$
\begin{aligned}
& \mathbf{B}_{n}^{(L) \triangleq[}\left[\begin{array}{cccc}
b_{\bmod (n, 16)} & b_{\bmod (n-1,16)} & \ldots & b_{\bmod (n-L+1,16)} \\
b_{\bmod (n+1,16)} & b_{\bmod (n, 16)} & \ldots & b_{\bmod (n-L+2,16)} \\
\vdots & \vdots & \ddots & \vdots \\
b_{\bmod (n+15,16)} & b_{\bmod (n+14,16)} & \ldots & b_{\bmod (n-L+16,16)}
\end{array}\right] \text { (9) } \\
& \mathbf{h}_{o} \triangleq\left[h\left(-L_{1} T_{\text {sam }}\right) h\left(\left(-L_{1}+1\right) T_{\text {sam }}\right) \ldots\right. \\
& \left.h\left(\left(L_{e}-L_{1}+1\right) T_{\text {sam }}\right)\right]^{T},
\end{aligned}
$$

and $\mathbf{w}_{n}$ is a vector containing the noise samples with a covariance matrix $\sigma_{w}^{2} \mathbf{I}_{N}\left(\mathbf{I}_{N}\right.$ being the $N \times N$ identity matrix). Similarly, the first received-signal vector for the cyclic prefix is given by

$$
\mathbf{r}_{n}=\mathbf{G}_{0}^{\left(L_{e}\right)} \mathbf{h}_{o}+\mathbf{w}_{n} \quad \text { for } n=10 N-L_{1}
$$

\footnotetext{
${ }^{1}$ Throughout this paper, the notations $(.)^{*},(.)^{T},(.)^{H}$ and $\|$.$\| stand for$
} conjugate, transpose, conjugate transpose and Euclidean norm, respectively. 
where

$$
\mathbf{G}_{0}^{(L)} \triangleq\left[\begin{array}{ccccc}
g_{0} & b_{15} & b_{14} & \ldots & b_{16-L+1} \\
g_{1} & g_{0} & b_{15} & \ldots & b_{16-L+2} \\
g_{2} & g_{1} & g_{0} & \ldots & b_{16-L+3} \\
\vdots & \vdots & \vdots & \ddots & \vdots \\
g_{15} & g_{14} & g_{13} & \ldots & g_{16-L}
\end{array}\right]
$$

For notational simplicity, we write $\mathbf{B}_{n}^{(L)}$ and $\mathbf{G}_{0}^{(L)}$ as $\mathbf{B}_{n}$ and $\mathbf{G}_{0}$, respectively, in the subsequent derivations.

\section{SYMBOL SYNCHRONIZATION PERFORMANCE CRITERION}

Having established the system model, the next question is how to define the "beginning" of an OFDM symbol. For frequency flat fading channels, the starting position is obvious and well defined. For Rician fading channel, it is reasonable to define the symbol boundary with respect to the first path. However, in a Rayleigh multipath fading channel (e.g., the one shown in Fig. 2), the channel contains some small taps at the beginning and the starting position of the channel is not clear. It can be defined as the first non-zero tap of the channel, as the first tap with energy larger than a certain threshold, as the position of the strongest path or any other definition. Because of this, the symbol boundary of a received OFDM symbol is not well defined. Even if we choose one of the above definitions as the reference position, there is no guarantee that a certain synchronization algorithm giving estimates close to the reference position would provide good performance in OFDM systems. Moreover, in OFDM systems, due to the existence of cyclic prefix, some timing offset can be tolerated as long as the samples within the FFT window are influenced by only one transmitted OFDM symbol. Therefore, the criterion that the synchronization error has to be within certain limits of a fixed reference point is not an appropriate performance measure for OFDM systems in frequency selective fading channels.

A more general and meaningful performance measure is the loss in system performance due to the synchronization error. With reference to Fig. 3, if the fast Fourier transform (FFT) window starts at position $n_{\varepsilon}$, the signal at the sub-carrier $k$ after the FFT operation, $z_{k}$, can be described as [19]

$$
z_{k}=e^{j 2 \pi\left(k / N_{F F T}\right) n_{\varepsilon}} \alpha\left(n_{\varepsilon}\right) a_{k} H_{k}+I_{k}+\eta_{k},
$$

where $a_{k}, H_{k}$ and $\eta_{k}$ are the data, channel transfer function and noise sample at sub-carrier $k$, respectively, $N_{F F T}$ is the number of FFT points in the OFDM system, which is 64 in IEEE 802.11a, $\alpha\left(n_{\varepsilon}\right)$ is the attenuation caused by the synchronization error, which can be well approximated by [19]

$$
\alpha\left(n_{\varepsilon}\right)=\sum_{i}\left|h\left(i T_{\text {sam }}\right)\right|^{2} \frac{N_{F F T}-\Delta \varepsilon_{i}}{N_{F F T}}
$$

where

$$
\Delta \varepsilon_{i} \triangleq \begin{cases}n_{\varepsilon}-i & n_{\varepsilon}>i \\ i-N-n_{\varepsilon} & n_{\varepsilon}<-(N-i) \\ 0 & \text { otherwise }\end{cases}
$$

and $I_{k}$ is the ISI plus inter-carrier interference (ICI) term at sub-carrier $k$ caused by the timing offset, which can be well approximated by Gaussian noise with power [19]

$$
\sigma_{\varepsilon}^{2}\left(n_{\varepsilon}\right)=\sum_{i}\left|h\left(i T_{s a m}\right)\right|^{2}\left(2 \frac{\Delta \varepsilon_{i}}{N_{F F T}}-\left(\frac{\Delta \varepsilon_{i}}{N_{F F T}}\right)^{2}\right) .
$$

For a particular channel realization, the signal-to-interferenceplus-noise ratio (SINR) is given by

$$
\operatorname{SINR}\left(n_{\varepsilon}\right)=\frac{\alpha^{2}\left(n_{\varepsilon}\right) \mathbb{E}\left[\left|a_{k} H_{k}\right|^{2}\right]}{\sigma_{\varepsilon}^{2}\left(n_{\varepsilon}\right)+\sigma_{w}^{2}} .
$$

Notice that for the special case that the equivalent channel length is smaller than the length of cyclic prefix (i.e., $L_{e}<N$ ) and $L_{e}-N \leq n_{\varepsilon} \leq 0$, then $\sigma_{\varepsilon}^{2}=0$, implying there is no ISI and ICI. On the other hand, if the length of the equivalent channel is larger than the length of the cyclic prefix, then $\sigma_{\varepsilon}^{2}>0$, therefore some ISI and ICI occur. In this case, the best we can do is to find a value of $n_{\varepsilon}$ which maximize the SINR. Noting that, due to (13), the SINR expression can be rewritten as

$$
\operatorname{SINR}\left(n_{\varepsilon}\right)=\frac{\mathbb{E}\left[\left|z_{k}\right|^{2}\right]-\sigma_{\varepsilon}^{2}\left(n_{\varepsilon}\right)-\sigma_{w}^{2}}{\sigma_{\varepsilon}^{2}\left(n_{\varepsilon}\right)+\sigma_{w}^{2}} .
$$

Since $\mathbb{E}\left[\left|z_{k}\right|^{2}\right]$ is a constant, maximizing SINR is equivalent to minimizing $\sigma_{\varepsilon}^{2}\left(n_{\varepsilon}\right)$. Therefore, the "ideal" symbol synchronizer should select $n_{\varepsilon}$ such that $\sigma_{\varepsilon}^{2}\left(n_{\varepsilon}\right)$ in (16) is minimized.

In practice, the ideal symbol synchronizer is not realizable since it requires the perfect knowledge of the channel realization. However, the ideal symbol synchronizer can serve as a reference to other practical synchronization algorithms. For a particular realization of channel, let $n_{\varepsilon}$ be the start of FFT window estimated by a particular symbol synchronization algorithm and $n_{i d}$ be that from the ideal symbol synchronizer. Then the loss of SINR, defined as the ratio of SINR obtained from the ideal symbol synchronizer to that from the non-ideal symbol synchronizer is given by

$$
\operatorname{SINR}_{l o s s}\left(n_{\varepsilon}\right) \triangleq \frac{\operatorname{SINR}_{i d}}{\operatorname{SINR}\left(n_{\varepsilon}\right)}=\frac{\alpha^{2}\left(n_{i d}\right)\left[\sigma_{\varepsilon}^{2}\left(n_{\varepsilon}\right)+\sigma_{w}^{2}\right]}{\alpha^{2}\left(n_{\varepsilon}\right)\left[\sigma_{\varepsilon}^{2}\left(n_{i d}\right)+\sigma_{w}^{2}\right]} .
$$

For a good symbol synchronization algorithm, the loss in SINR with respect to the ideal synchronizer should be very small. Similar to [5], we define a synchronization failure as the event that the loss in SINR is greater than a tolerable system degradation. That is,

$$
P_{f}(\Delta \gamma) \triangleq P\left(10 \log _{10}\left(\operatorname{SINR}_{\text {loss }}\right)>\Delta \gamma\right),
$$

where $P_{f}(\Delta \gamma)$ is the probability of synchronization failure given that the tolerable system degradation (in $\mathrm{dB}$ ) is $\Delta \gamma$, and $P($.) denotes the probability of an event.

\section{PROPOSED SYMBOL SYNCHRONIZATION ALGORITHM}

For the packet structure shown in Fig. 1, determining the FFT window position for the OFDM data symbols actually involves two major steps. The first one is to identify a reference position (e.g., the transition form the short training symbol to the cyclic prefix of the long training symbol in the middle 
of the preamble) such that all the subsequent transitions can be predicted. We refer this step as frame synchronization. The second step is to locate the FFT window position within an OFDM symbol such that the ISI plus ICI introduced is minimized, which we refer it as symbol synchronization. Notice that in some cases, frame synchronization and symbol synchronization are the same process. For example, if the channel is frequency flat or the multipath channel is causal and with total length smaller than the cyclic prefix of the OFDM symbols, then the optimum position for the FFT window is at $n_{\varepsilon}=0$ [20] and symbol synchronization follows naturally and immediately once frame synchronization is achieved. However, for the channel shown in Fig. 2, which is non-causal and the total length may be larger than the cyclic prefix of the OFDM symbols, symbol synchronization is essential.

\section{A. Frame synchronization}

Suppose that the arrival of the preamble can be identified by detecting the received-signal energy (e.g., using the methods in [8] or [12]), the problem of detecting the transition between the short training symbols and the cyclic prefix of the long training symbols can be decomposed into two sub-problems. Let $\mathbf{r}_{n_{1}}$ be a received-signal vector within the short training interval. Since $\mathbf{r}_{n_{1}}$ may not align with the beginning of a short training symbol, the first sub-problem is to determine the current time offset with respect to the last short training symbol and hence predict the starting time of the next (expected) short training symbol. Suppose this starting time is denoted by the time index $n_{2}$. After $n_{2}$ is obtained, the second sub-problem is to examine the incoming vectors $\mathbf{r}_{n_{2}+q N}, q=0,1,2, \ldots, 10$, and to determine if they belong to a short training symbol or a cyclic prefix of the long training symbol. The transition point is declared at the time instant that the first $\mathbf{r}_{n_{2}+q N}$ belongs to the cyclic prefix.

1) First stage: Assume that the received-signal vector $\mathbf{r}_{n_{1}}$ is $i_{o}$ samples $\left(i_{o} \in\{0,1, \ldots, 15\}\right)$ offset from the beginning of the current short training symbol. The probability density function (PDF) of the received-signal vector $\mathbf{r}_{n_{1}}$ is

$$
p\left(\mathbf{r}_{n_{1}} ; \boldsymbol{\theta}, i, L\right)=\frac{1}{\pi^{N} \sigma^{2 N}} \exp \left\{-\frac{1}{\sigma^{2}}\left\|\mathbf{r}_{n_{1}}-\mathbf{B}_{i} \mathbf{h}\right\|^{2}\right\},
$$

where $^{2} \boldsymbol{\theta} \triangleq\left[\Re\left(\mathbf{h}^{T}\right), \Im\left(\mathbf{h}^{T}\right), \sigma^{2}\right]^{T}$ with $\mathbf{h}, \sigma^{2}$ and $L$ being the trial values of $\mathbf{h}_{o}, \sigma_{w}^{2}$ and $L_{e}$, respectively. Note that $\mathbf{B}_{i}$ depends on $i$ and $L$ (see (9)). It is not possible to jointly estimate $\mathbf{h}_{o}, \sigma_{w}^{2}, i_{o}$ and $L_{e}$ by directly maximizing $p\left(\mathbf{r}_{n_{1}} ; \boldsymbol{\theta}, i, L\right)$ since (apart from the fact that the implementation complexity would be extremely high) the largest possible $L$ is always chosen $[17, \mathrm{p} .223]$. One criterion that gets around this problem is the generalized ML rule [17, p.223], in which we maximize

$$
\Psi\left(\mathbf{r}_{n_{1}} ; i, L\right)=\ln p\left(\mathbf{r}_{n_{1}} ; \hat{\boldsymbol{\theta}}, i, L\right)-\frac{1}{2} \ln \operatorname{det}(\mathbf{I}(\hat{\boldsymbol{\theta}} \mid i, L)),
$$

where the second term is a penalty term that becomes more negative as $L$ increases. In the above expression, $\hat{\boldsymbol{\theta}}$ is the ML

${ }^{2}$ Notations $\Re($.$) and \Im($.$) denote the real part and imaginary part, respec-$ tively. estimate of $\theta$ [16, p.186] [17, p.222] (given $i$ and $L$ ) and $\mathbf{I}(\boldsymbol{\theta} \mid i, L)$ is the Fisher information matrix [16, p.525] of $\boldsymbol{\theta}$ (given $i$ and $L$ ).

It can be proved that [21], the generalized ML rule (22) can be simplified to

$$
\begin{aligned}
& \Psi_{1}\left(\mathbf{r}_{n_{1}} ; i, L\right)= \\
& \quad(-N+L+1) \ln \left\|\mathbf{r}_{n_{1}}-\mathbf{B}_{i}\left(\mathbf{B}_{0}^{H} \mathbf{B}_{0}\right)^{-1} \mathbf{B}_{i}^{H} \mathbf{r}_{n_{1}}\right\|^{2}-\xi(L)
\end{aligned}
$$

where $\xi(L) \triangleq L \ln 2+\ln \left(\operatorname{det}\left(\mathbf{B}_{0}^{H} \mathbf{B}_{0}\right)\right)$ is a function of $L$ only and can be pre-computed and stored in a look-up table to reduce the real-time computational complexity. The firststage synchronization algorithm, which jointly estimates the effective channel order $L_{e}$ and the delay $i_{o}$ from the receivedsignal vector $\mathbf{r}_{n_{1}}$, becomes

$$
\hat{i}, \hat{L}=\arg \max _{\substack{i \in\{0,1, \ldots, 15\}, L \in\left\{1,2, \ldots, L_{\max }\right\}}} \Psi_{1}\left(\mathbf{r}_{n_{1}} ; i, L\right)
$$

where $L_{\max }$ is the maximum possible value of the channel order. The starting position of the next expected short training symbol is then given by $n_{2}=n_{1}+16-\hat{i}$.

As discussed in Section III, since the starting position of a frame is not well defined in multipath Rayleigh fading channels, it is necessary to clarify what is the meaning of estimated offset by maximizing (22). Let $\{\hat{i}, \hat{L}\}$ be the set of values that maximize (22), then $\hat{i}$ is the number of offset samples from the beginning of the current short training symbol conditioned that the number of paths of the channel is $\hat{L}$ and the offset is with respect to the first estimated path. Notice that the generalized ML criterion (22) tends to ignore the channel paths of small energy, therefore, $\hat{L}<L_{e}$. For example, in the channel shown in Fig. 2, the estimated channel length from the generalized ML criterion is $\hat{L}=8$ $(-2 \leq i \leq 5)$ at $\mathrm{SNR}=30 \mathrm{~dB}$.

2) Second stage: The second step is to determine the smallest value of $q$ such that $\mathbf{r}_{n_{2}+q N}$ belongs to the cyclic prefix. This problem can be handled by Neyman-Pearson (NP) detection approach [17, ch.3]. Let $H_{g}$ and $H_{b}$ be the hypotheses that $\mathbf{r}_{n_{2}+q N}$ belongs to the cyclic prefix and the short training symbol, respectively. In each test, the probability that the received-signal vector belongs to the short training symbol is the same as the probability that it belongs to the cyclic prefix. It follows that $n_{2}+q N$ is the point of transition if the condition

$$
p\left(\mathbf{r}_{n_{2}+q N} \mid H_{g}\right)>p\left(\mathbf{r}_{n_{2}+q N} \mid H_{b}\right)
$$

occurs for the first time, where

$$
\begin{aligned}
& \left.p\left(\mathbf{r}_{n} \mid H_{g}\right)=\frac{1}{\pi^{N} \sigma^{2 N}} \exp \left\{-\frac{1}{\sigma^{2}}\left\|\mathbf{r}_{n}-\mathbf{G}_{0} \mathbf{h}\right\|^{2}\right\}\right]_{L=\hat{L}} \\
& \left.p\left(\mathbf{r}_{n} \mid H_{b}\right)=\frac{1}{\pi^{N} \sigma^{2 N}} \exp \left\{-\frac{1}{\sigma^{2}}\left\|\mathbf{r}_{n}-\mathbf{B}_{0} \mathbf{h}\right\|^{2}\right\}\right]_{L=\hat{L}}
\end{aligned}
$$

Taking logarithm on both sides of (25), putting $\widehat{\mathbf{h}}=\left(\mathbf{G}_{0}^{H} \mathbf{G}_{0}\right)^{-1} \mathbf{G}_{0}^{H} \mathbf{r}_{n_{2}+q N}$ into (26) and $\widehat{\mathbf{h}}=$ 
$\left(\mathbf{B}_{0}^{H} \mathbf{B}_{0}\right)^{-1} \mathbf{B}_{0}^{H} \mathbf{r}_{n_{2}+q N}$ into (27), we find that $n_{2}+q N$ is the point of transition if

$$
\Psi_{2}\left(\mathbf{r}_{n_{2}+q N} \mid H_{g}\right)>\Psi_{2}\left(\mathbf{r}_{n_{2}+q N} \mid H_{b}\right)
$$

where

$$
\begin{aligned}
& \left.\Psi_{2}\left(\mathbf{r}_{n} \mid H_{g}\right)=\mathbf{r}_{n}^{H} \mathbf{G}_{0}\left(\mathbf{G}_{0}^{H} \mathbf{G}_{0}\right)^{-1} \mathbf{G}_{0}^{H} \mathbf{r}_{n}\right]_{L=\hat{L}} \\
& \left.\Psi_{2}\left(\mathbf{r}_{n} \mid H_{b}\right)=\mathbf{r}_{n}^{H} \mathbf{B}_{0}\left(\mathbf{B}_{0}^{H} \mathbf{B}_{0}\right)^{-1} \mathbf{B}_{0}^{H} \mathbf{r}_{n}\right]_{L=\hat{L}} .
\end{aligned}
$$

\section{B. Position of FFT window}

After the transition between short training symbols and the cyclic prefix of the long training symbols is detected, we can predict that the beginning of the first data carrying OFDM symbol (the OFDM symbol for the signal field) is $n_{3} \triangleq n_{2}+$ $\hat{q} N+(32+2 \times 64)$, where $32+2 \times 64$ is the number of samples for the long training symbols. If the equivalent channel has exactly $\hat{L}$ paths, then the allowable range for the starting point of the FFT window is $\left\{n_{3}+\hat{L}, \ldots, n_{3}+N\right\}$. However, in reality, it is expected that there are some channel taps with small energy preceding and following the $\hat{L}$ paths. With the observation that the "head" and "tail" of the equivalent channel have energy die down more or less at the same rate, it is reasonable to set the start of the FFT window at

$$
n_{4} \triangleq n_{3}+\hat{L}+\lfloor(N-\hat{L}) / 2\rfloor \text {. }
$$

For subsequent OFDM symbols, the starting points of the FFT window are then $n_{4}+\ell\left(N+N_{F F T}\right)$ where $\ell$ is a positive integer.

Remark 2. It is interesting to note that the form of the generalized ML rule in (23) is similar to the GAIC used in [14]. However, there are several differences between the algorithm proposed here and the one in [14]. First, our proposed algorithm uses the short training symbols and the cyclic prefix of the long training symbol to achieve frame synchronization whereas the algorithm in [14] uses only the long training symbol. Second, the observation-vector length used in the proposed algorithm is 16 whereas it is 64 for the one in [14]. Third, our proposed algorithm is developed based on a time-domain approach while a frequency-domain analysis is employed in [14]. Lastly, our proposed algorithm considers a more general channel model than the algorithm in [14] (see Remark 1 of Section II-A). Performance and complexity comparisons between the proposed algorithm and the technique of [14] are provided in the next section.

\section{Simulation RESUlts AND Discussions}

Simulations are run in order to investigate the synchronization performance of the proposed algorithm. Due to space limitation, only limited results are presented in this paper. More results can be found in [21]. The received samples are generated according to (6) with $L_{1}=15$ and $L_{e}=36$ so that the range of index $i$ in (6) is $\{-15, \ldots, 20\}$. This enables the equivalent channel to be accurately represented (see Fig. 2 ). The channels are generated using the same parameters as that of Fig. 2. The channel is fixed during each packet but independent from one packet to another. $L_{\max }$ is assumed to be $12 . n_{1}$ was treated as a uniform random variable over $[5 N+1,6 N]$ in the simulation, and a value of $n_{1}$ was randomly generated in each run. For each simulation run, the loss of SINR is calculated using (19), where the ideal symbol synchronizer selects a starting point for the FFT window such that (16) is minimized. The noise samples are i.i.d., zero mean complex Gaussian random variable. Each point of result is obtained by averaging over $10^{4}$ Monte-Carlo runs.

The algorithm based on GAIC [14] is designed to detect the transition between $g(t)$ and $c(t)$ in the preamble. Due to the fact that the GAIC algorithm provides also an estimate of the channel length, the FFT window starting position for the first OFDM data symbol can be calculated in a similar way to the proposed algorithm (see (31)). That is, the FFT window starts at $\hat{n}_{G A I C}+2 \times 64+\hat{L}_{G A I C}+\left\lfloor\left(N-\hat{L}_{G A I C}\right) / 2\right\rfloor$ where $\hat{n}_{G A I C}$ and $\hat{L}_{G A I C}$ are the timing estimate and channel length estimate from the GAIC algorithm, respectively.

Fig. 4 shows the probability of synchronization failure $P_{f}(0.5 \mathrm{~dB})$ as a function of SNR for the proposed algorithm and the algorithm based on GAIC. It is obvious that both algorithms have similar performances. Furthermore, the probability of failures for both algorithms are very small. Notice that the curves of $P_{f}$ in general show an "U shape". This is because at low SNRs, the estimation is not accurate due to the high level of noise, while at high SNRs, although the estimated positions can be quite accurate, a small amount of shift with respect to the ideal position leads to a large amount of loss in SINR (see (19)).

Finally, we want to mention that although the performances of the proposed algorithm is comparable to that of GAIC algorithm, the complexity of the proposed algorithm is much smaller. This can be explained as follows. Both algorithms involve a least-squares fitting in the form of $\|\mathbf{r}-\mathbf{\Phi r}\|^{2}$, where $\mathbf{r}$ is an observation vector in the time domain for the proposed algorithm and is an observation vector transformed into the frequency domain using FFT for the algorithm based on GAIC, $\boldsymbol{\Phi}$ is some square matrix depending on the parameters to be estimated (compare (23) of this paper with (4) in [14]). Since the observation length is only 16 for the proposed algorithm while it is 64 for the GAIC algorithm, the number of multiplications for the proposed algorithm in each hypothesis test is 16 times less than that of GAIC algorithm. Taking into account the fact that, for the proposed algorithm, the number of hypothesis tests is smaller than that of the GAIC algorithm, and there is no need to transform the observation vector into frequency domain before least-squares fitting, the proposed algorithm is at least 16 times less complex than the GAIC algorithm.

\section{CONCLUSIONS}

In this paper, based on the maximum-likelihood principle and the preamble structure of IEEE 802.11a standard, a new symbol synchronization algorithm for IEEE 802.11a WLANs 
over frequency-selective fading channels is proposed. A realistic channel model was employed, which includes the effects of the physical channel, filtering and unknown sampling phase offset. Loss in system performance due to synchronization error was used as a performance criterion. Computer simulations showed that the proposed algorithm exhibits comparable performance to the algorithm based on GAIC, but the proposed algorithm requires significantly reduced complexity.

\section{REFERENCES}

[1] IEEE Computer Society, IEEE Std 802.11a-1999, 30 Dec. 1999.

[2] R. van Nee and R. Prasad, OFDM for Wireless Multimedia Communications, Boston: Artech House Publishers, 2000.

[3] J. Heiskala and J. Terry, OFDM Wireless LANs: A Theoretical and Practical Guide, Sams Publishing, 2002.

[4] M. Speth, F. Classen and H. Meyr, "Frame synchronization of OFDM systems in frequency selective fading channels," Proc. IEEE VTC'97, pp. 1807-1811, May 1997.

[5] M. Speth, D. Daecke and H. Meyr, "Minimum overhead burst synchronization for OFDM based broadband transmission," Proc. Globecom 98, pp. 3227-3232, 1998.

[6] J.-J. van de Beek, M. Sandell and P. O. Borjesson, "ML estimation of time and frequency offset in OFDM systems," IEEE Trans. Signal Processing, vol. 45, pp. 1800-1805, Jul. 1997.

[7] D. Lee and K. Cheun, "Coarse symbol synchronization algorithms for OFDM systems in multipath channels," IEEE Commun. Letters, vol. 6, pp. 446-448, Oct. 2002.

[8] T. M. Schmidl and D. C. Cox, "Robust frequency and timing synchronization for OFDM," IEEE Trans. Commun., vol. 45, pp. 1613-1621, Dec. 1997.

[9] S H. Muller-Weinfurtner, "On the optimality of metrics for coarse frame synchronization in OFDM: a comparison," Proc. PIMRC 98, pp. 533537, 1998.

[10] M. Mizoguchi, T. Onizawa and T. Kumagai, H. Takanashi and M. Morikura, "A fast burst synchronization scheme for OFDM" Proc. IEEE ICUPC 98, pp. 125-129, 1998.

[11] T. Keller, L. Piazzo, P. Mandarini and L. Hanzo, "Orthogonal frequency division multiplex synchronization techniques for frequency-selective fading channels," IEEE J. Select. Areas Commun., vol. 19, pp. 999-1008, June 2001.

[12] A. J. Coulson, "Maximum likelihood synchronization for OFDM using a pilot symbol: algorithms," IEEE J. Select. Areas Commun., vol. 19, pp. 2486-2494, Dec. 2001.

[13] K.-W. Yip, T.-S. Ng and Y.-C. Wu, "Impacts of multipath fading on the timing synchronization of IEEE 802.11a wireless LANs," Proc. ICC 2002, pp. 517-521, Apr. 28 - May 2, 2002.

[14] E. G. Larsson, G. Liu, J. Li and G. B. Giannakis, "Joint symbol timing and channel estimation for OFDM based WLANs," IEEE Commun. Letters, vol. 5, pp. 325-327, Aug. 2001.

[15] J. G. Proakis, Digital Communications, 3rd ed., New York: McGrawHill, 1995.

[16] S. M. Kay, Fundamentals of Statistical Signal Processing, Vol. 1: Estimation Theory, New York: Prentice-Hall, 1993.

[17] S. M. Kay, Fundamentals of Statistical Signal Processing, Vol. 2: Detection Theory, New York: Prentice-Hall, 1998.

[18] H. Meyr, M. Oerder and A. Polydoros, "On sampling rate, analog prefiltering, and sufficient statistics for digital receivers," IEEE Trans. Commun., vol. 42, pp. 3208-3213, Dec. 1994. 5modulation," IEEE Trans. Commun., vol. 45, pp. 1327-1337, Oct. 1997.

[19] M. Speth, S. A. Fechtel, G. Fock and H. Meyr, "Optimum receiver design for wireless broad-band systems using OFDM - Part I," IEEE Trans. Commun., vol. 47, pp. 1668-1677, Nov. 1999.

[20] H. Steendam and M. Moeneclaey, "Analysis and optimization of the performance of OFDM on frequency-selective time-selective fading channel," IEEE Trans. Commun., vol. 47, pp. 1811-1819, Dec. 1999.

[21] Y.-C. Wu, K.-W. Yip, T.-S. Ng and E. Serpedin, "Maximum-Likelihood Symbol Synchronization for IEEE 802.11a WLANs on Unknown Frequency-Selective Fading Channels," accepted in IEEE Trans. Wireless Commun.

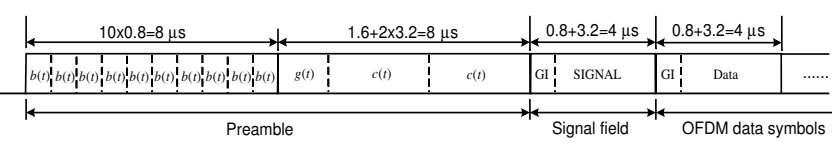

$b(t)$ : short training symbol

$g(t):$ cyclic prefix of $c(t)$

$c(t)$ : long training symbol

Fig. 1. Packet structure for IEEE 802.11a WLANs.

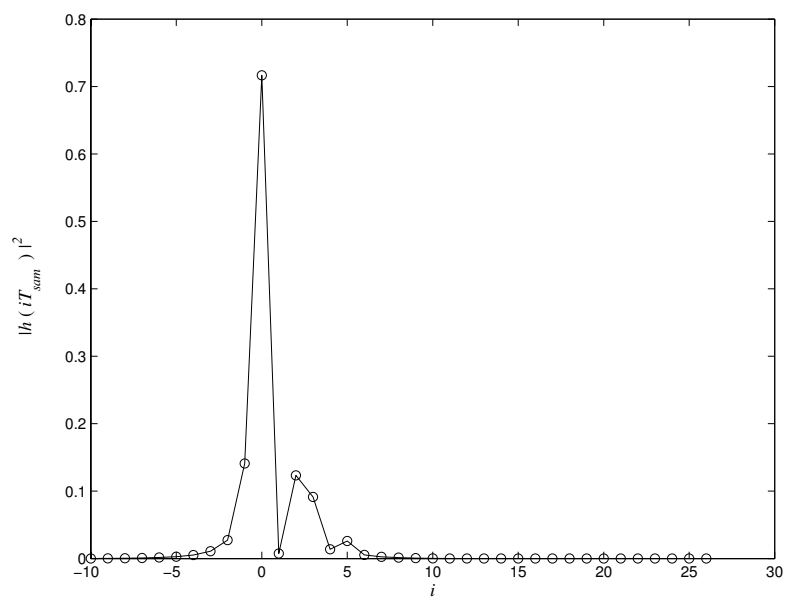

Fig. 2. An example of $\left|h\left(i T_{\text {sam }}\right)\right|^{2}$.

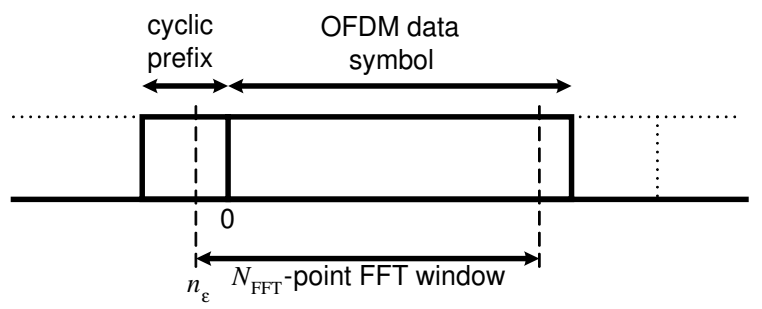

Fig. 3. OFDM symbol and FFT position.

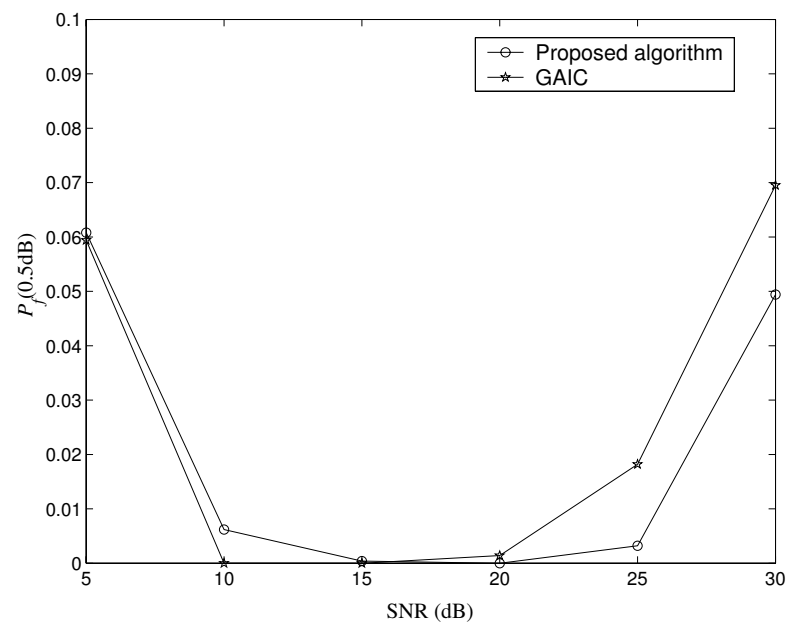

Fig. 4. $\quad P_{f}(0.5 \mathrm{~dB})$ for the proposed algorithm and algorithm based on GAIC [14] as a function of SNR. 